\title{
Fatty fish consumption and risk of latent autoimmune diabetes in adults
}

\author{
JE Löfvenborg ${ }^{1}$, T Andersson ${ }^{1,2}$, P-O Carlsson ${ }^{3}$, M Dorkhan $^{4}$, L Groop ${ }^{4}$, M Martinell ${ }^{5}$, T Tuomi $^{6}$, A Wolk ${ }^{1}$ and S Carlsson ${ }^{1}$
}

\begin{abstract}
OBJECTIVE: It has been suggested that intake of fatty fish may protect against both type 1 and type 2 diabetes. Hypotheses rest on the high marine omega-3 fatty acid eicosapentaenoic acid+docosahexaenoic acid (EPA+DHA) and vitamin D contents, with possible beneficial effects on immune function and glucose metabolism. Our aim was to investigate, for the first time, fatty fish consumption in relation to the risk of latent autoimmune diabetes in adults (LADA).

METHODS: Analyses were based on data from a Swedish case-control study with incident cases of LADA ( $n=89)$ and type 2 diabetes $(n=462)$ and randomly selected diabetes-free controls $(n=1007)$. Diabetes classification was based on the onset of age $(\geqslant 35)$, glutamic acid decarboxylase autoantibodies, and C-peptide. A validated food frequency questionnaire was used to derive information on previous intake of fish, polyunsaturated long-chain omega-3 fatty acids ( $n-3$ PUFA) and supplementation of fish oil and vitamin D. Odds ratios (ORs) with 95\% confidence intervals (Cls) were calculated using logistic regression, adjusted for age, gender, body mass index (BMI), family history of diabetes, physical activity, smoking, education, and consumption of alcohol, fruit, vegetables and red meat.

RESULTS: Weekly fatty fish consumption ( $\geqslant 1$ vs $<1$ serving per week), was associated with a reduced risk of LADA but not type 2 diabetes (OR 0.51, 95\% Cl 0.30-0.87, and 1.01, 95\% Cl 0.74-1.39, respectively). Similar associations were seen for estimated intake of n-3 PUFA ( $\geqslant 0.3 \mathrm{~g}$ per day; LADA: OR 0.60, 95\% Cl 0.35-1.03, type 2 diabetes: OR 1.14, 95\% Cl 0.79-1.58) and fish oil supplementation (LADA: OR 0.47, 95\% Cl 0.19-1.12, type 2 diabetes: OR 1.58, 95\% Cl 1.08-2.31).

CONCLUSIONS: Our findings suggest that fatty fish consumption may reduce the risk of LADA, possibly through effects of marineoriginated omega-3 fatty acids.
\end{abstract}

Nutrition \& Diabetes (2014) 4, e139; doi:10.1038/nutd.2014.36; published online 20 October 2014

\section{INTRODUCTION}

Recent findings suggest that fatty fish consumption may be protective against autoimmune diabetes in children, ${ }^{1,2}$ a Norwegian study found a reduced risk of type 1 diabetes in children who were given dietary supplementation of cod liver oil during first year of life. ${ }^{1}$ Likewise, a US study showed that the intake of omega-3 fatty acids, was inversely associated with islet autoimmunity in genetically susceptible children. ${ }^{2}$ Fish intake has also been associated with a reduced risk of other autoimmune diseases in adults such as rheumatoid arthritis ${ }^{3}$ and multiple sclerosis. ${ }^{4}$ An inverse relationship between fatty fish consumption and type 2 diabetes has also been proposed ${ }^{5}$ but as shown in two recent reviews, findings for type 2 diabetes are inconclusive. ${ }^{6,7}$

The hypothesized effect has primarily been attributed to the polyunsaturated long-chain omega-3 fatty acids ( $n-3$ PUFA) found in fish and fish oil, especially eicosapentaenoic (EPA) and docosahexaenoic (DHA) acids. EPA and DHA possess antiinflammatory, immunomodulatory and gene expression regulatory properties. ${ }^{8}$ Owing to the highly unsaturated properties of EPA and DHA, they are able to be rapidly incorporated in the membranes of immune cells, thereby alter their function ${ }^{8}$ and thus may be beneficial with regard to autoimmune diseases. This cell membrane incorporation happens partly on the expense of the pro-inflammatory arachidonic acid. ${ }^{8}$ The anti-inflammatory property may be of importance for autoimmune diabetes but also type 2 diabetes as both conditions have been associated with lowgrade inflammation, ${ }^{9}$ further, these properties of EPA and DHA have been suggested also to affect insulin signaling. ${ }^{10}$ Fatty fish is also an important dietary source of vitamin $D$, which in recent years has attained much attention for its immunoregulatory properties and inverse association with both type 1 and type 2 diabetes. $^{11}$

Latent autoimmune diabetes in adults (LADA) may be the second most common form of diabetes, ${ }^{12}$ accounting for $9 \%$ of all diabetes in Europe according to a recent report. LADA has been described as a hybrid between type 1 and type 2 diabetes; ${ }^{13}$ Like type 1 diabetes, it is an autoimmune form of diabetes, but with a slower autoimmune process than in classic type 1 diabetes. In addition, LADA patients tend to have features of type 2 diabetes including overweight and insulin resistance. ${ }^{14}$ Risk factors are largely unexplored and as far as we know, the risk of LADA in relation to intake of fatty fish has never been investigated before.

Our aim was to investigate the risk of LADA, and type 2 diabetes in relation to consumption of fatty fish and dietary supplementation of fish oil and vitamin D using data from Epidemiologic study of risk factors for LADA and type 2 diabetes (ESTRID) - a new Swedish population-based study with incident cases.

\footnotetext{
${ }^{1}$ Institute of Environmental Medicine, Karolinska Institutet, Stockholm, Sweden; ${ }^{2}$ Center for Occupational and Environmental Medicine, Stockholm County Council, Stockholm, Sweden; ${ }^{3}$ Department of Medical Sciences, Uppsala University, Uppsala, Sweden; ${ }^{4}$ Department of Clinical Sciences, Lund University, Malmö, Sweden; ${ }^{5}$ Department of Public Health and Caring Sciences, Uppsala University, Uppsala, Sweden and ${ }^{6}$ Division of Endocrinology, Department of Medicine, Helsinki University Central Hospital; Research Program for Diabetes and Obesity, University of Helsinki and Folkhalsan Research Center, Helsinki, Finland. Correspondence: Dr S Carlsson, Institute of Environmental Medicine, Karolinska Institutet, Box 210, Stockholm SE171 77, Sweden.

E-mail: sofia.carlsson@ki.se

Received 3 July 2014; revised 18 August 2014; accepted 2 September 2014
} 


\section{MATERIALS AND METHODS}

Study population and design

Results were based on data from ESTRID; (http://www.ki.se/imm/estrid), initiated in 2010 (Figure 1). The ESTRID data collection has been described in detail elsewhere. ${ }^{15}$ In short, incident cases of diabetes were identified continuously using two population-based diabetes registries in Sweden (ANDIS; All New Diabetics in Scania http://andis.ludc.med.lu.se/, and ANDiU; All New Diabetics in Uppsala http://www.andiu.se/), aimed at characterizing all new diabetes cases with regard to clinical and genetic factors. To ESTRID we invited all new cases of LADA identified in Skåne (from 2010 onwards) and Uppsala (from 2012 onwards) together with a random sample of patients with newly diagnosed type 2 diabetes (four per LADA case). For each LADA case, six diabetes-free controls ( $\geqslant 35$ years) were randomly selected from the population of Scania and Uppsala, using the Swedish population registry. They were matched to the cases by time and region (incidence density sampling). ${ }^{16}$ Analyses in the present paper were based on data collected in ESTRID between its initiation in September 2010 and July 2013, with a response rate of $80 \%$ (cases) and 67\% (controls). As the present investigation concerned dietary habits prior to diagnosis, the analysis of this paper were based on all patients participating in ESTRID within 6 months of diagnosis, including 89 cases of LADA, 462 cases of type 2 diabetes, and 1007 controls. The study was approved by the Regional Ethical Review Board at Karolinska Institutet, Sweden, and all participants provided written informed consent.

\section{Diabetes classification and clinical analysis}

Cases were diagnosed within the health-care system and at diagnosis, blood samples were collected and analysed at the central laboratories of the university hospitals of each county. Glutamic acid decarboxylase autoantibodies was analyzed with enzyme-linked immuno sorbent assay and levels are reported as an index value in relation to standard serum. The method gives a maximum value of $250 \mathrm{IU} \mathrm{ml}^{-1}$. At a cutoff of $10.7 \mathrm{IU} \mathrm{ml} \mathrm{m}^{-1}$, sensitivity was $84 \%$ and specificity $98 \% .{ }^{17}$ For the current investigation, and to minimize false positive cases, levels $>20 \mathrm{IU} \mathrm{ml}^{-1}$ were regarded as positive, in line with the diabetes classification used in the ANDIS registry. C-peptide was measured by an IMMULITE 2000 (Siemens Healthcare Diagnostics Product Ltd, Llanberis, UK) or by Cobas e 601 analyzer (Roche Diagnostics, Mannheim, Germany). ${ }^{18}$ LADA was diagnosed if age $\geqslant 35$ years, glutamic acid decarboxylase autoantibodies $>20 \mathrm{U} \mathrm{ml}^{-1}$ and C-peptide $\geqslant 0.2 \mathrm{nmolI}^{-1}$ (Immulite) or $\geqslant 0.3 \mathrm{nmolI}^{-1}$ (Cobas). Type 2 diabetes criteria were age $\geqslant 35$ years, glutamic acid decarboxylase autoantibodies $<10$ and C-peptide $>0.6 \mathrm{nmolI}^{-1}$ (Immulite) or $>0.72$ $\mathrm{nmol} \mathrm{I}^{-1}$ (Cobas). This was in line with previously used criteria, ${ }^{13}$ except for C-peptide levels which replaced the commonly used insulin criteria to distinguish LADA from classical type 1 diabetes with adult onset. This is a more direct indicator of remaining insulin production and a slow 'latent onset' than mode of treatment, which is open to subjective evaluation. Insulin resistance (HOMA-IR) and $\beta$-cell function (HOMA- $\beta$ ) was assessed by HOMA (homeostatic model assessment), based on the relationship between fasting glucose and C-peptide levels. ${ }^{19}$

\section{Assessment of exposures and covariates}

Exposure data was obtained from a comprehensive health and lifestyle questionnaire including a 132-item food frequency questionnaire. This food frequency questionnaire has been validated against diet diaries ${ }^{20-23}$ and for some items including fish intake, against biological markers. ${ }^{24}$ There were 11 seafood items, four regarded fatty fish (herring/Baltic herring/mackerel, salmon, sardines, smoked fish), four asked about lean fish (cod/pollock/plaice/blue hake, tuna, pike/pike perch/perch, fish fingers) and the remainder about shell fish, roe and other fish types (than those mentioned above). There were also nine questions asking about red meat products, five about fresh fruit/berries, and 15 about vegetables. The Spearman correlation coefficients between these items and four 1-week weighted diet records ranged from 0.6 to 0.7 for fruits, from 0.4 to 0.6 for vegetables (AW, unpublished data, 1992) and were 0.5 for fatty fish, 0.4 for lean fish and 0.6 for other seafood. ${ }^{23}$ Participants were asked how often, on average during the past year, they had consumed each item, with eight pre-defined response alternatives ranging from 0 times per month to $\geqslant 3$ times per day. Patients were specifically instructed to provide information on dietary habits as they had been the year preceding their diagnosis. Average daily consumption of fatty fish was estimated by converting the responses for each item to average daily intake and then summing these into one fatty fish variable. Average daily marine n-3 PUFA (EPA+DHA) intake from diet was calculated by multiplying the intake frequency of each type of fish or seafood product with nutrient data from the Swedish National Food Agency database. The estimated n-3 PUFA intake based on the questionnaire has been validated against fatty acid content in subcutaneous adipose tissue, with a Pearson's correlation of $0.41 .^{23}$ The questionnaire also included information on intake of vitamins, minerals and supplements, including fish oil supplements and vitamin D. Participants were instructed to report if they have used any of the

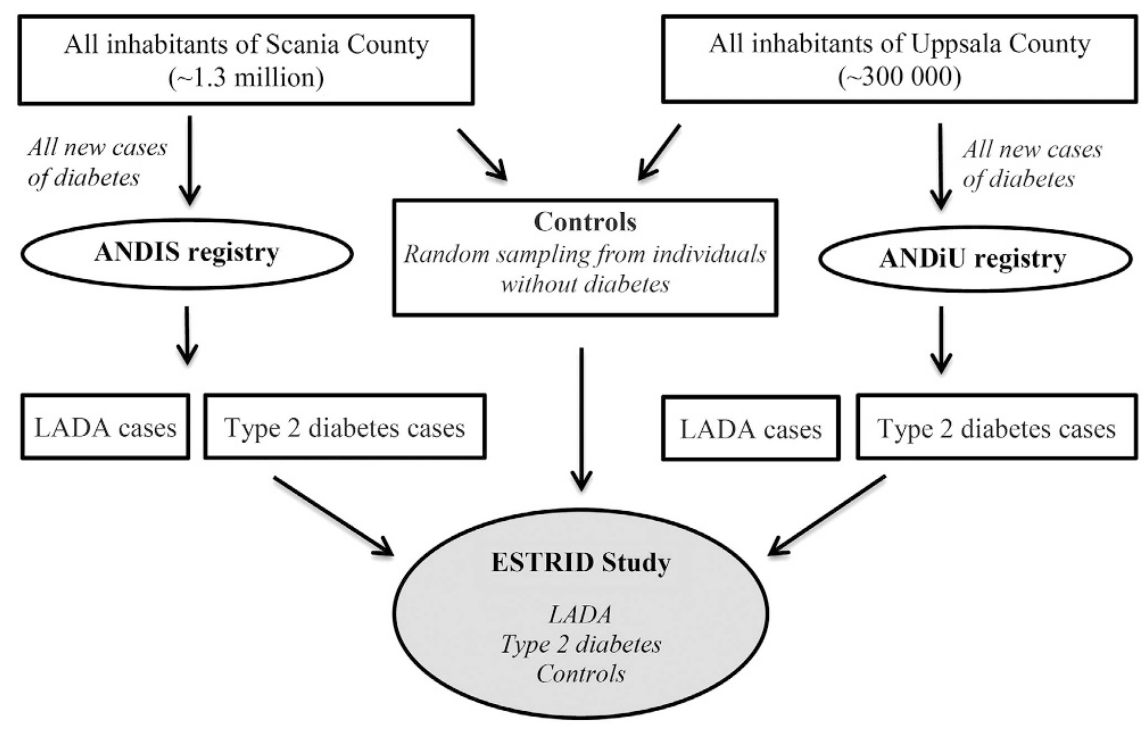

Questionnaire data on health and lifestyle factors (cases and controls)

Clinical data on GAD autoantibodies, C-peptide, time of diagnosis, treatment, genetic data (cases)

Figure 1. Schematic of the ESTRID study design. 
products on a regular basis for a minimum of 3 months and if so, to specify the time period of use. Consumption of supplements was rare and therefore, consumption of fish oil and vitamin D was categorized as never/ ever in the analysis.

We also collected information on potential confounders, including height and weight used for calculation of BMI $\left(\mathrm{kg} \mathrm{m}^{-2}\right)$, education, smoking status, physical activity, family history of diabetes, and consumption of alcohol.

\section{Statistical analysis}

Odds ratios (OR) with 95\% confidence intervals (Cl) of LADA and type 2 diabetes were calculated in relation to fatty fish intake, fish oil supplementation and vitamin D supplementation using conditional logistic regression models (SAS 9.3; SAS Institute Inc.). If the Cls did not include 1.0, the ORs were interpreted as significant (this corresponds to a double-sided $P$-value $<0.05$ ). Models were run adjusted for age and gender (model 1 ), age, gender, education level $(\geqslant 9,10-12,>12$ years in school), smoking (never, ever), alcohol consumption (abstainer, former drinker, 0.1-4.8 g per week, $\geqslant 4.9 \mathrm{~g}$ per week), physical activity (sedentary, moderate, moderate regular, regular), family history of diabetes, and BMI ( $\mathrm{kg} \mathrm{m}^{-2}$ ) (model 2). To account for dietary habits, additional adjustment was made for intake of red meat products, vegetables and fresh fruit/berries (model 3). Results from model 3 are presented in the text unless otherwise stated. $P$-values were calculated using $X^{2}$ (proportions) and Wilcoxon-Mann-Whitney test (means).

\section{RESULTS}

Mean age was 59.2 years in controls, 57.6 years in LADA patients and 62.9 years in type 2 diabetes patients. Compared with type 2 diabetes patients, those with LADA were leaner, had lower levels of C-peptide, HOMA- $\beta$ and HOMA-IR (Table 1). Fatty fish intake was lowest among LADA patients, $51.7 \%$ consumed less than one serving per week compared with $37.4 \%$ of type 2 diabetes patients and $38.5 \%$ of controls ( $P=0.0150$, LADA vs controls). Fish oil supplementation tended to be less frequent among LADA patients; $7.4 \%$ reported ever use compared with $16.1 \%$ of controls $(P=0.0636)$. Among controls, $21.2 \%$ had ever used vitamin $\mathrm{D}$ and/ or fish oil supplementation compared with $12.4 \%$ of LADA patients $(P=0.0487)$.

In the analysis adjusted for age and gender, we observed a reduced risk of LADA associated with weekly consumption of fatty fish (OR 0.56, 95\% Cl 0.36-0.88) (Table 2), whereas no association was found for type 2 diabetes (OR 0.93 , 95\% Cl 0.73-1.19) (Table 3). The reduced risk of LADA remained virtually unaffected by additional adjustment for BMl, education, physical activity, smoking, alcohol consumption, family history of diabetes, red meat, vegetables and fresh fruit/berries (OR $0.51,95 \% \mathrm{Cl} 0.30$ 0.87, whereas no association with type 2 diabetes could be detected (OR 1.01, 95\% Cl 0.74-1.39). More frequent consumption than 1-2 times per week did not further reduce the risk. Consumption of lean fish was neither associated with LADA (OR 0.99, 95\% Cl 0.56-1.72) nor type 2 diabetes (OR 0.87, 95\% $\mathrm{Cl}$ 0.63-1.19).

There was also indication of an inverse association between estimated dietary marine n-3 PUFA levels and LADA (Table 2); OR was estimated at $0.60(95 \% \mathrm{Cl} 0.35-1.03)$ for intakes of $\geqslant 0.3 \mathrm{~g}$ per day (approximately equivalent to $\geqslant 1$ serving of fatty fish per week). No further risk reduction was seen for intakes of $>0.6 \mathrm{~g}$ per day and no association was seen between n-3 PUFA and type 2 diabetes (Table 3). Use of fish oil supplements showed a similar tendency toward a reduced risk of LADA (OR 0.47, 95\% Cl 0.19 1.12), but no indication of a reduced risk for type 2 diabetes (OR $1.58,95 \% \mathrm{Cl} 1.08-2.31$ ). Additional adjustment for fatty fish intake did not change these risk estimates. There was a tendency toward a reduced risk of both LADA and type 2 diabetes in consumers of vitamin D supplements, but numbers were small.

\section{DISCUSSION}

Our findings indicate that intake of fatty fish may reduce the risk of LADA. We are not aware of any previous studies on autoimmune diabetes in adults in relation to fatty fish but these results are in line with studies on type 1 diabetes in children ${ }^{1,2}$ as well as with other autoimmune diseases in adults, namely rheumatoid arthritis and multiple sclerosis. ${ }^{3,4}$ A proposed mechanism underlying the association is beneficial effects of marine $n-3$ PUFAs including EPA and DHA on the immune system, including improved membrane fluidity of immune cells and alterations in gene expression. ${ }^{8}$ Our findings were also compatible with a protective effect of vitamin $D$, previously shown to be inversely associated with type 1 diabetes. ${ }^{25}$ Several different cell types of the immune system display vitamin $D$ receptors including $T$ cells and $B$ cells and the active metabolite of vitamin $D, 1,25(\mathrm{OH})_{2} D$, has the ability to regulate proliferation and function of these immune cells. One commonly suggested mechanism for the involvement of vitamin $\mathrm{D}$ in incident type 1 diabetes includes modification of $\mathrm{T}$ cell differentiation. ${ }^{26}$ The reduced risk of LADA was seen at intakes of $\geqslant 1$ serving per week, or marine $n-3$ PUFA intakes of $\geqslant 0.3 \mathrm{~g}$ per day, with no further risk reduction at higher levels, suggesting a threshold effect of dietary marine n-3 PUFA. Similar findings were seen in a study of rheumatoid arthritis where constant

Table 1. Characteristics of subjects included in the analyses

\begin{tabular}{|c|c|c|c|c|c|}
\hline Characteristics & Controls & Type 2 diabetes & $\angle A D A$ & P-value ${ }^{a}$ & $\mathrm{P}$-value $\mathrm{b}$ \\
\hline No. of individuals & 1007 & 462 & 89 & & \\
\hline Age, mean, years (s.d.) & $59.0(13.3)$ & $62.9(10.4)$ & $57.6(13.1)$ & 0.0002 & 0.3615 \\
\hline Men, \% & 47.3 & 59.3 & 48.3 & 0.0547 & 0.8498 \\
\hline BMI, mean, $\mathrm{kg} \mathrm{m}^{-2}$ (s.d.) & $26.0(4.1)$ & $31.4(5.8)$ & $27.1(4.5)$ & $<0.0001$ & 0.0188 \\
\hline 1st degree relatives with diabetes (\%) & $24.3 \%$ & $45.0 \%$ & $48.3 \%$ & 0.5679 & $<0.0001$ \\
\hline GADA, median, IU ml ${ }^{-1}$ (interquartile range) & - & - & $250^{c}(199)$ & - & \\
\hline C-peptide, mean, nmol I-1 (s.d.) & - & $1.33(0.62)$ & $0.68(0.46)$ & $<0.0001$ & \\
\hline HOMA- $\beta$, mean (s.d.) & - & $4.75(3.38)$ & $2.04(2.02)$ & $<0.0001$ & \\
\hline HOMA-IR, mean (s.d.) & - & $0.67(0.43)$ & $0.39(0.27)$ & $<0.0001$ & \\
\hline$<1$ serving of fatty fish per week, $\%$ & 38.5 & 37.5 & 51.7 & 0.012 & 0.0150 \\
\hline Fish oil supplementation, ever, \% & 16.5 & 19.1 & 9.0 & 0.022 & 0.0636 \\
\hline Vitamin D supplementation, ever, \% & 8.2 & 4.8 & 4.50 & 0.91 & 0.2099 \\
\hline Fish oil or/and vitamin D, ever, \% & 21.2 & 22.3 & 12.4 & 0.0341 & 0.0487 \\
\hline
\end{tabular}

Abbreviations: BMI, body mass index; GADA, glutamic acid decarboxylase autoantibodies; HOMA- $\beta$, homeostatic model assessment- $\beta$-cell function; HOMA-IR, homeostatic model assessment-insulin resistance; LADA, latent autoimmune diabetes in adults. ${ }^{\text {a }}$-value for difference between LADA and type 2 diabetes. ${ }^{\mathrm{b}} P$-value for difference between LADA and controls. ${ }^{\mathrm{C}} \mathrm{GAD}$ autoantibody levels were truncated at 250. 
Table 2. Odds ratios of LADA in relation to intake of fatty fish, EPA and DHA from seafood and fish oil and vitamin D supplementation

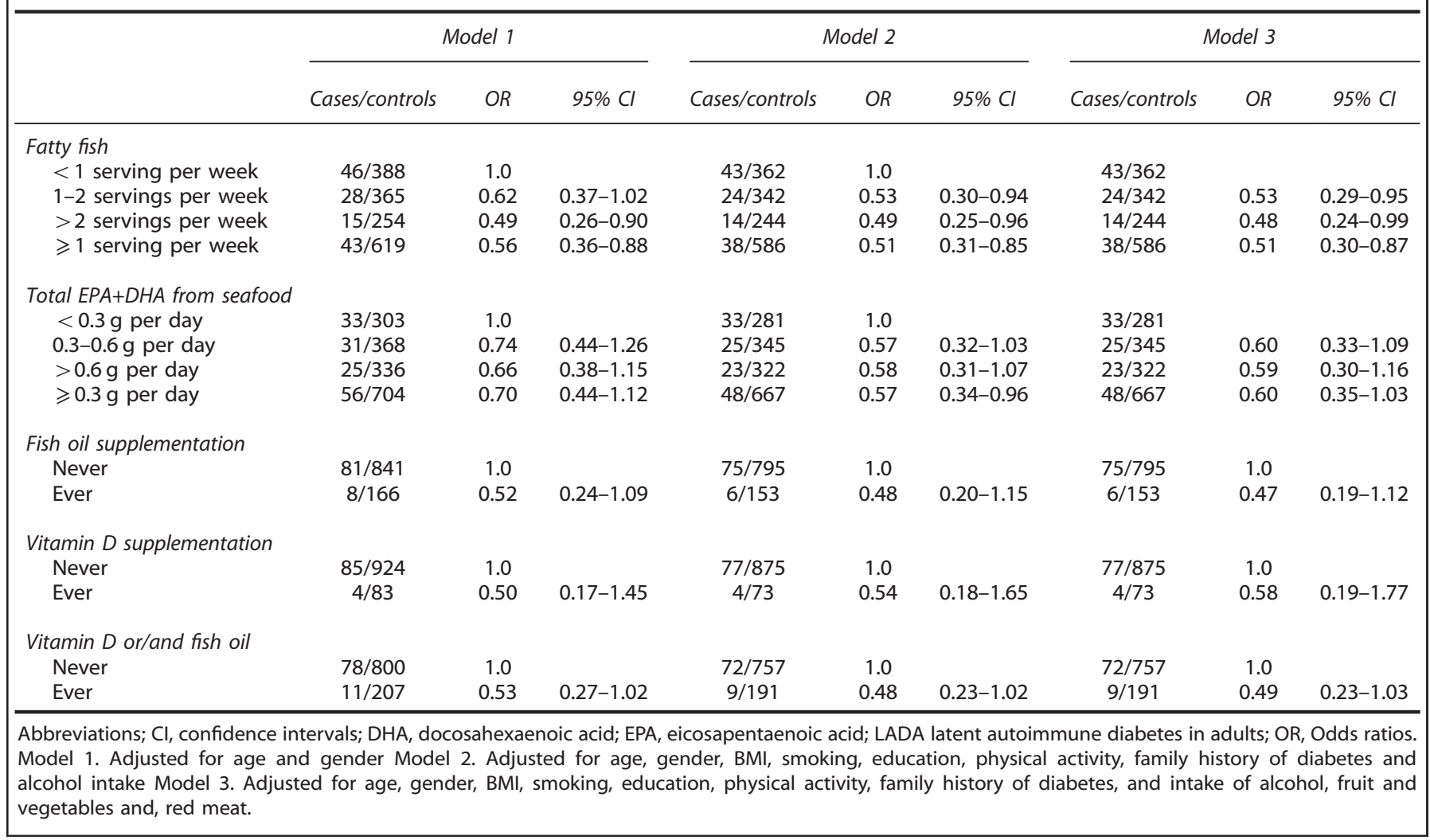

Table 3. Odds ratios of Type 2 diabetes in relation to intake of fatty fish, EPA and DHA and supplementation of vitamin D and fish oil

\begin{tabular}{|c|c|c|c|c|c|c|c|c|c|}
\hline & Cases/controls & $O R$ & $95 \% \mathrm{Cl}$ & Cases/controls & $O R$ & $95 \% \mathrm{Cl}$ & Cases/controls & $O R$ & $95 \% \mathrm{Cl}$ \\
\hline$<1$ serving per week & $173 / 388$ & 1 & & $157 / 362$ & 1 & & $157 / 362$ & & \\
\hline $1-2$ servings per week & $168 / 365$ & 0.95 & $0.72-1.24$ & $157 / 342$ & 1.10 & $0.78-1.54$ & $157 / 342$ & 1.04 & $0.73-1.46$ \\
\hline$>2$ servings per week & $121 / 254$ & 0.91 & $0.68-1.23$ & $117 / 244$ & 1.08 & $0.74-1.58$ & $117 / 244$ & 0.97 & $0.65-1.45$ \\
\hline \multicolumn{10}{|l|}{ Total EPA+DHA from seafood } \\
\hline$<0.3 \mathrm{~g}$ per day & $130 / 303$ & 1.0 & & $116 / 281$ & & & $116 / 281$ & & \\
\hline $0.3-0.6 \mathrm{~g}$ per day & $176 / 368$ & 1.08 & $0.81-1.44$ & $166 / 345$ & 1.22 & $0.85-1.77$ & $166 / 345$ & 1.17 & $0.81-1.71$ \\
\hline$>0.6 \mathrm{~g}$ per day & $156 / 336$ & 0.98 & $0.73-1.32$ & $149 / 322$ & 1.16 & $0.79-1.70$ & $149 / 322$ & 1.03 & $0.68-1.55$ \\
\hline$\geqslant 0.3 \mathrm{~g}$ per day & $332 / 704$ & 1.03 & $0.80-1.33$ & $315 / 667$ & 1.19 & $0.85-1.67$ & $315 / 667$ & 1.14 & $0.79-1.58$ \\
\hline \multicolumn{10}{|l|}{ Fish oil supplementation } \\
\hline Ever & 22/83 & 0.79 & $0.44-1.43$ & $21 / 73$ & 0.79 & $0.41-1.50$ & $21 / 73$ & 0.75 & $0.39-1.43$ \\
\hline \multicolumn{10}{|l|}{ Vitamin D or/and fish oil } \\
\hline Never & $365 / 800$ & 1 & & $97 / 207$ & 1 & & $97 / 207$ & 1 & \\
\hline Ever & $97 / 207$ & 1.01 & $0.82-1.47$ & $93 / 191$ & 1.40 & $0.97-2.01$ & 93/191 & 1.35 & $0.93-1.94$ \\
\hline
\end{tabular}


relative risks were observed for intakes of $>0.35 \mathrm{~g}$ per day. ${ }^{3}$ Furthermore, a threshold effect at $0.25 \mathrm{~g}$ per day was observed in a pooled analysis of randomized trials and prospective studies investigating the role of fish and fish oil intake on mortality from coronary heart disease. ${ }^{27}$

In confirmation of most previous European reports, ${ }^{6}$ there was no association between fatty fish consumption and type 2 diabetes. Furthermore, an increased risk of type 2 diabetes was seen in users of fish oil supplementation. Our findings are compatible with a reduced risk of type 2 diabetes in users of vitamin D supplementation as shown previously, ${ }^{11}$ although number of users was small. Vitamin $D$ has been proposed to influence the pathophysiology of type 2 diabetes by means of affecting $\beta$ cell function and insulin action. The pancreatic $\beta$ cells may be affected though binding of $1,25(\mathrm{OH})_{2} \mathrm{D}$ to its vitamin $\mathrm{D}$ receptors, or by activation of vitamin $D$ within the $\beta$ cells. Insulin action may be influenced by stimulating the expression of insulin receptors, leading to improved glucose transportation. ${ }^{28}$

Dietary information was based on a validated food frequency questionnaire, and the estimated marine n-3 PUFA levels from the questionnaire show a fairly strong correlation with PUFA content in fat tissue. ${ }^{23} \mathrm{~A}$ limitation of the case-control study design is the retrospective collection of potential exposure; that is, cases respond to the questionnaire after diagnosis, but while only information on habits prior to diagnosis is of relevance. Hence, if patients increased their consumption of fatty fish after recommendations from their physicians, and reported accordingly, this would lead to overestimation of the fatty fish intake among cases. To minimize this bias, we provided careful instructions that dietary habits should be reported as they were the year preceding diagnosis, and furthermore, we only included patients who responded to the questionnaire within 6 months of diagnosis. Importantly an increase in fish intake following diagnosis does not explain why LADA patients consumed half as much fatty fish and fish oil supplements as controls and patients with type 2 diabetes; overestimation of fish intake in patients would lead to an overestimation of the OR rather than the opposite. The association could be due to underreporting of fish consumption among patients, but this does not explain the different results seen for LADA and type 2 diabetes. In this context it is noteworthy that with regard to type 2 diabetes, our results were in line with previous European reports based on prospective data with fish intake measured several years prior to disease onset. ${ }^{6}$

With regard to confounding, fatty fish could be an indicator of a healthy lifestyle or diet associated with a low diabetes risk; for example, fatty fish consumers may consume less red meat, known to be associated with an increased risk of type 2 diabetes, ${ }^{29}$ or more fruit and vegetables, associated with a reduced risk of type 2 diabetes. ${ }^{30}$ Notably, the ORs remained virtually unaffected by adjustment for a number of potential confounders, including education, lifestyle and dietary factors. Also, to explain an OR of the magnitude reported in this study, the factor must be a strong correlate of fatty fish intake as well as a strong risk factor of LADA, but not type 2 diabetes. The sensitivity and specificity of the glutamic acid decarboxylase autoantibodies to distinguish autoimmune diabetes implies that we may have some false positive cases among the LADA patients; that is, patients with type 2 diabetes and vise versa. This misclassifications is however unlikely to explain the different results seen for the two diabetes types. Non-response could introduce bias if participating controls differ from the population that generated the cases with regard to dietary habits. ${ }^{16}$ For example, subjects with high socioeconomic status, known to have healthier eating habits, were overrepresented in the sample, which could lead to an overestimation of the inverse association between fatty fish intake and LADA. We have no reason to believe this is the case as the educational level of controls in ESTRID were in line with those in the general population of Scania, based on data from statistics Sweden (www.scb.se).

In summary, our findings suggest that fatty fish consumption may protect against LADA, possibly through effects of marine n-3 PUFA on the immune system. No association was observed between fatty fish and type 2 diabetes indicating that the association with LADA is driven by other mechanisms than those related to type 2 diabetes features. Numbers were small and our findings may be due to chance. Still, the internal and external $^{1-4}$ consistency of findings and the magnitude of the potential effect implies that the issue of fatty fish and fish oil in relation to autoimmune diabetes is an important topic to elucidate further.

\section{CONFLICT OF INTEREST}

The authors declare no conflict of interest.

\section{ACKNOWLEDGEMENTS}

The ESTRID study is supported by The Swedish Research Council, The Swedish Research Council for Health, Working Life and Welfare, AFA Insurance, and The Swedish Diabetes Association. The Swedish Research Council is also supporting ANDIS (including ALF funding for clinical research in medicine) and ANDiU (including EXODIAB - strategic governmental funding for excellence of diabetes research in Sweden).

\section{AUTHOR CONTRIBUTIONS}

TA contributed to the statistical approaches and considerations, interpretations of data, and reviewing of the manuscript. P-OC, MD, LG, and MM contributed to collection and interpretation of data and reviewing of the manuscript. $\Pi$ and AW contributed with interpretation of data and reviewing of the manuscript. SC contributed to the design and conduct of the study, interpretation of data and writing of the manuscript. JEL contributed to the conception, design and conduct of the study, interpretation of data, and writing of the manuscript. All authors approved the final version of the manuscript. JEL is the guarantor, affirming the integrity of the data and that this is a truthful report of the study.

\section{REFERENCES}

1 Stene LC, Joner G. the Norwegian Childhood Diabetes Study Group Use of cod liver oil during the first year of life is associated with lower risk of childhood-onset type 1 diabetes: a large, population-based, case-control study. Am J Clin Nutr 2003; 78: 1128-1134.

2 Norris JM, Yin X, Lamb MM, Barriga K, Seifert J, Hoffman M et al. Omega-3 polyunsaturated fatty acid intake and islet autoimmunity in children at increased risk for type 1 diabetes. JAMA 2007; 298: 1420-1428.

3 Di Giuseppe D, Wallin A, Bottai M, Askling J, Wolk A. Long-term intake of dietary long-chain n-3 polyunsaturated fatty acids and risk of rheumatoid arthritis: a prospective cohort study of women. Ann Rheum Dis 2013; doi:10.1136/ annrheumdis-2013-203338.

4 Bäärnhielm M, Olsson T, Alfredsson L. Fatty fish intake is associated with decreased occurrence of multiple sclerosis. Mult Scler 2014; 20: 726-732.

5 Zhang M, Picard-Deland E, Marette A. Fish and marine omega-3 polyunsaturated fatty acid consumption and incidence of type 2 diabetes: a systematic review and meta-analysis. Int J Endocrinol 2013; 2013: 501015.

6 Patel PS, Forouhi NG, Kuijsten A, Schulze MB, van Woudenbergh GJ, Ardanaz E et al. InterAct Consortium. The prospective association between total and type of fish intake and type 2 diabetes in 8 European countries: EPIC-InterAct Study. Am J Clin Nutr 2012; 95: 1445-1453.

7 Wallin A, Di Giuseppe D, Orsini N, Patel PS, Forouhi NG, Wolk A. Fish consumption, dietary long-chain n-3 fatty acids, and risk of type 2 diabetes: systematic review and meta-analysis of prospective studies. Diabetes Care 2012; 35: 918-929.

8 Calder PC. n-3 Fatty acids, inflammation and immunity: new mechanisms to explain old actions. Proc Nutr Soc 2013; 72: 326-336.

9 Duncan BB, Schmidt MI, Pankow JS, Ballantyne CM, Couper D, Vigo A et al. Atherosclerosis risk in communities study. Low-grade systemic inflammation and the development of type 2 diabetes: the atherosclerosis risk in communities study. Diabetes 2003; 52: 1799-1805.

10 Risérus U, Willett WC, Hu FB. Dietary fats and prevention of type 2 diabetes. Prog Lipid Res 2009; 48: 44-51. 
11 Wolden-Kirk H, Overbergh L, Christensen HT, Brusgaard K, Mathieu C. Vitamin D and diabetes: its importance for beta cell and immune function. Mol Cell Endocrinol 2011; 347: 106-120.

12 Hawa Ml, Kolb H, Schloot N, Beyan H, Paschou SA, Buzzetti R et al. Adult-onset autoimmune diabetes in Europe in prevalent with a broad clinical phenotype. Diabetes Care 2013; 36: 908-913.

13 Tuomi T, Santoro N, Caprio S, Cai M, Weng J, Groop L. The many faces of diabetes: a disease with increasing heterogeneity. Lancet 2014; 383: 1084-1094.

14 Chiu HK, Tsai EC, Juneja R, Stoever J, Brooks-Worrell B, Goel A et al. Equivalent insulin resistance in latent autoimmune diabetes in adults (LADA) and type 2 diabetic patients. Diabetes Res Clin Pract 2007; 77: 237-244.

15 Löfvenborg JE, Andersson T, Carlsson PO, Dorkhan M, Groop L, Martinell M et al. Coffee consumption and the risk of latent autoimmune diabetes in adults results from a Swedish case-control study. Diabetic Med 2014; 31: 799-805.

16 Vandenbroucke JP, Pearce N. Case-control studies: basic concepts. Int J Epidemiol 2012; 41: 1480-1489.

17 Rahmati K, Lernmark A, Becker C, Foltyn-Zadura A, Larsson K, Ivarsson SA et al. A comparison of serum and EDTA plasma in the measurement of glutamic acid decarboxylase autoantibodies (GADA) and autoantibodies to islet antigen-2 (IA-2A) using the RSR radioimmunoassay (RIA) and enzyme linked immunosorbent assay (ELISA) kits. Clin Lab 2008; 54: 227-235.

18 Smolcic VS, Bilic-Zulle L, Fisic E. Validation of methods performance for routine biochemistry analytes at Cobas 6000 analyzer series module c501. Biochem Med (Zagreb) 2011; 21: 182.

19 Levy JC, Matthews DR, Hermans MP. Correct homeostasis model assessment (HOMA) evaluation uses the computer program. Diabetes Care 1998; 21 2191-2192.

20 Levitan EB, Westgren CW, Liu S, Wolk A. Reproducibility and validity of dietary glycemic index, dietary glycemic load, and total carbohydrate intake in 141 Swedish men. Am J Clin Nutr 2007; 85: 548-553.

21 Messerer M, Johansson SE, Wolk A. The validity of questionnaire-based micronutrient intake estimates is increased by including dietary supplement use in Swedish men. J Nutr 2004; 134: 1800-1805.
22 Khani BR, Ye W, Terry P, Wolk A. Reproducibility and validity of major dietary patterns among Swedish women assessed with a food-frequency questionnaire. J Nutr 2004; 134: 1541-1545.

23 Wallin A, Di Giuseppe D, Burgaz A, Håkansson N, Cederholm T, Michaëlsson K et al. Validity of food frequency questionnaire-based estimates of long-term long-chain n-3 polyunsaturated fatty acid intake. Eur J Nutr 2014; 53: 549-555.

24 Wolk A, Larsson SC, Johansson JE, Ekman P. Long-term fatty fish consumption and renal cell carcinoma incidence in women. JAMA 2006; 296: 1371-1376.

25 Hyppönen E. Vitamin D and increasing incidence of type 1 diabetes-evidence for an association? Diabetes Obes Metab 2010; 12: 737-743.

26 Bikle DD. Vitamin D regulation of immune function. Vitam Horm 2011; 86: 1-21.

27 Mozaffarian D, Rimm EB. Fish intake, contaminants, and human health: evaluating the risks and the benefits. JAMA 2006; 296: 1885-1899.

28 Pittas AG, Lau J, Hu FB, Dawson-Hughes B. The role of vitamin D and calcium in type 2 diabetes. A systematic review and meta-analysis. J Clin Endocrinol Metab 2007; 92: 2017-2029.

29 Pan A, Sun Q, Bernstein AM, Manson JE, Willett WC, Hu FB. Changes in red meat consumption and subsequent risk of type 2 diabetes mellitus: three cohorts of US men and women. JAMA Intern Med 2013; 173: 1328-1335.

30 Carter P, Gray LJ, Troughton J, Khunti K, Davies MJ. Fruit and vegetable intake and incidence of type 2 diabetes mellitus: systematic review and meta-analysis. BMJ 2010; 341: c4229.

(c) (1) This work is licensed under a Creative Commons Attributioncc. Non Commercial-ShareAlike 4.0 International License. The images or other third party material in this article are included in the article's Creative Commons license, unless indicated otherwise in the credit line; if the material is not included under the Creative Commons license, users will need to obtain permission from the license holder to reproduce the material. To view a copy of this license, visit http:// creativecommons.org/licenses/by-nc-sa/4.0/ 